\title{
An improved local error estimator for symmetric time-stepping schemes ${ }^{1}$
}

\author{
Winfried Auzinger \\ Technische Universität Wien, Institut für Analysis und Scientific Computing, Wiedner Hauptstrasse 8-10/E101, A-1040 Wien, Austria \\ Othmar Koch \\ Universität Wien, Institut für Mathematik, Oskar-Morgenstern-Platz 1, A-1090 Wien, Austria
}

\begin{abstract}
We propose a symmetrized version of the defect to be used in the estimation of the local time-stepping error of symmetric one-step methods for the time propagation of linear autonomous evolution equations. Using the anticommutator of the numerical flow and the right-hand side operator in the definition of the defect of the numerical approximation, a local error estimator is obtained which has higher accuracy asymptotically than an established version using the common defect. This theoretical result is illustrated for a splitting method applied to a linear Schrödinger equation.
\end{abstract}

Keywords: Numerical time integration, one-step methods, symmetric schemes, local error estimation

2010 MSC: 65L05, 65L50

\section{Introduction}

Consider the evolution equation

$$
u^{\prime}(t)=H u(t), \quad u(0)=u_{0}
$$

defined on a Banach space $\mathcal{X}$, with a generally unbounded time-independent operator $H: \mathcal{D}(H) \subset \mathcal{X} \rightarrow \mathcal{X}$ which generates a semigroup. We assume that the problem is well-defined with a sufficiently regular solution $u$, and denote the fundamental solution by $\mathcal{E}=\mathcal{E}(t)=\mathrm{e}^{t H}$, such that

$$
\mathcal{E}^{\prime}=H \mathcal{E}=\mathcal{E} H=\frac{1}{2} \mathcal{E} H+\frac{1}{2} H \mathcal{E}=\frac{1}{2}\{\mathcal{E}, H\}
$$

where $\{U, V\}=U V+V U$ denotes the anti-commutator of two operators. Observe that the symmetrized problem (2) is a special case of a Sylvester-type differential equation.

Let $\mathcal{S}(t)$ denote the numerical flow generated by a one-step integration scheme of order $p$, with $\mathcal{S} \approx \mathcal{E}$. The local error of the integrator is denoted by $\mathcal{L}=\mathcal{S}-\mathcal{E}$.

In computational practice, the error estimator $\tilde{\mathcal{L}} \approx \mathcal{L}$ discussed below will be applied in a finite-dimensional setting after (spatial) discretization of the given evolution equation and can serve as a reliable basis for the adaptive choice of optimal time step-sizes for efficient large-scale computations. In our reasoning below we tacitly assume all the appearing quantities to be as smooth as required.

\section{Defect-based local error estimation}

\subsection{Classical version}

We consider (w.l.o.g.) a single step of the one-step method represented by $\mathcal{S}$ starting from $t=0$. An asymptotically correct computable local error estimator based on the (classical) defect $\mathcal{D}_{c}$ of the numerical solution,

$$
\mathcal{D}_{c}(t)=\mathcal{S}^{\prime}(t)-H \mathcal{S}(t),
$$

\footnotetext{
${ }^{1}$ This manuscript version is made available under the CC-BY-NC-ND 4.0 license http://creativecommons.org/licenses/by-nc-nd/4.0/. The original publication is available from https://doi.org/10.1016/j.aml.2018.03.001.
} 
has been successfully employed particularly for the adaptive integration of time-dependent Schrödinger equations by splitting methods in a series of papers $[1,2,3]$. Here the local error is approximated on the basis of the relation

$$
\mathcal{L}(t)=\int_{0}^{t} \mathrm{e}^{(t-\tau) H} \mathcal{D}_{c}(\tau) \mathrm{d} \tau=\frac{1}{p+1} t \mathcal{D}_{c}(t)+\mathscr{O}\left(t^{p+2}\right) .
$$

This represents the approximation of the local error integral by Hermite quadrature, involving a single evaluation at $\tau=t$ and exploiting the fact that, due to convergence order $p$, the derivatives of the integrand vanish at $\tau=0$ up to order $p-1$. The resulting a posteriori error estimator is denoted by $\tilde{\mathcal{L}}_{c}(t):=\frac{1}{p+1} t \mathcal{D}_{c}(t)$.

\subsection{Symmetrized version}

Self-adjoint one-step schemes (also called time-reversible or symmetric) are characterized by the property $\mathcal{S}(-t) \mathcal{S}(t)=$ Id. We stress that self-adjoint schemes have even order $p$, see [4, Theorem II.3.2].

In the following we assume that $\mathcal{S}$ is self-adjoint, and thus the parameter $p$, denoting the order of the scheme, is an even integer. For this case, a modified construction employing a symmetrized defect,

$$
\mathcal{D}_{s}(t):=\mathcal{S}^{\prime}(t)-\frac{1}{2}\{\mathcal{S}(t), H\}
$$

yields a local error estimator with higher asymptotical accuracy, as we reason below. We will show that, in contrast to (4), we even have

$$
\mathcal{L}(t)=\int_{0}^{t} \mathrm{e}^{(t-\tau)\left(\frac{1}{2} H\right)} \mathcal{D}_{s}(\tau) \mathrm{e}^{(t-\tau)\left(\frac{1}{2} H\right)} \mathrm{d} \tau=\frac{1}{p+1} t \mathcal{D}_{s}(t)+\mathscr{O}\left(t^{p+3}\right) .
$$

The error estimator $\tilde{\mathcal{L}}_{s}(t):=\frac{1}{p+1} t \mathcal{D}_{s}(t)$ with symmetrized defect $\mathcal{D}_{s}$ is based on Hermite quadrature formula as for the classical version, but now applied to $(6)$.

Theorem 1. Consider a selfadjoint one-step scheme of (even) order $p \geq 2$, represented by $\mathcal{S}(t)$, applied to a linear evolution equation (1). Let $\mathcal{E}(t)=\mathrm{e}^{t H}$, and

$$
\widehat{\mathcal{S}}(t)=\mathcal{S}(t)-\tilde{\mathcal{L}}_{s}(t)=\mathcal{S}(t)-\frac{1}{p+1} t \mathcal{D}_{s}(t), \quad \mathcal{D}_{s}(t) \text { from }(5) .
$$

Then the local error operator $\widehat{\mathcal{L}}(t)=\widehat{\mathcal{S}}(t)-\mathcal{E}(t)$ of the corrected scheme represented by $\widehat{\mathcal{S}}(t)$ satisfies

$$
\widehat{\mathcal{L}}(t)=\mathscr{O}\left(t^{p+3}\right),
$$

i.e., the corrected scheme has even order $p+2$.

Proof: By assumption on $\mathcal{S}$ we have $\mathcal{S}(-t) \mathcal{S}(t)=I$. By construction of $\mathcal{D}_{s}$ we have $\mathcal{D}_{s}(t)=\mathscr{O}\left(t^{p}\right)$, and the corrected scheme is of order $p+1$ at least (cf. e.g. [2]). It will have even order $p+2$ if it is again selfadjoint or sufficiently close to selfadjoint. Thus, let us consider $\widehat{\mathcal{S}}(-t) \widehat{\mathcal{S}}(t)$ :

$$
\begin{aligned}
& \widehat{\mathcal{S}}(-t) \widehat{\mathcal{S}}(t)=\left(\mathcal{S}(-t)+\frac{1}{p+1} t \mathcal{D}_{s}(-t)\right)\left(\mathcal{S}(t)-\frac{1}{p+1} t \mathcal{D}_{s}(t)\right) \\
& =\underbrace{\mathcal{S}(-t) \mathcal{S}(t)}_{=I}-\frac{1}{p+1} t\left(\mathcal{S}(-t) \mathcal{D}_{s}(t)-\mathcal{D}_{s}(-t) \mathcal{S}(t)\right)-\frac{1}{(p+1)^{2}} t^{2} \mathcal{D}_{s}(-t) \mathcal{D}_{s}(t) .
\end{aligned}
$$

Here, $\mathcal{S}(-t) \mathcal{D}_{s}(t)-\mathcal{D}_{s}(-t) \mathcal{S}(t)$ is the critical term. With

$$
\begin{aligned}
\mathcal{D}_{s}(t) & =\mathcal{S}^{\prime}(t)-\frac{1}{2}\{\mathcal{S}(t), H\}, \\
\mathcal{D}_{s}(-t) & =\mathcal{S}^{\prime}(-t)-\frac{1}{2}\{\mathcal{S}(-t), H\}
\end{aligned}
$$

we have

$$
\begin{aligned}
\mathcal{S}(-t) \mathcal{D}_{s}(t) & =\mathcal{S}(-t) \mathcal{S}^{\prime}(t)-\mathcal{S}(-t) \frac{1}{2}\{\mathcal{S}(t), H\} \\
& =\mathcal{S}(-t) \mathcal{S}^{\prime}(t)-\frac{1}{2} H-\frac{1}{2} \mathcal{S}(-t) H \mathcal{S}(t),
\end{aligned}
$$


and

$$
\begin{aligned}
\mathcal{D}_{s}(-t) \mathcal{S}(t) & =\mathcal{S}^{\prime}(-t) \mathcal{S}(t)-\frac{1}{2}\{\mathcal{S}(-t), H\} \mathcal{S}(t) \\
& =\mathcal{S}^{\prime}(-t) \mathcal{S}(t)-\frac{1}{2} \mathcal{S}(-t) H \mathcal{S}(t)-\frac{1}{2} H .
\end{aligned}
$$

Together with $\mathcal{S}(-t) \mathcal{S}(t)=I$, whence

$$
0=\frac{\mathrm{d}}{\mathrm{d} t}(\mathcal{S}(-t) \mathcal{S}(t))=-\mathcal{S}^{\prime}(-t) \mathcal{S}(t)+\mathcal{S}(-t) \mathcal{S}^{\prime}(t),
$$

we obtain

$$
\mathcal{S}(-t) \mathcal{D}_{s}(t)-\mathcal{D}_{s}(-t) \mathcal{S}(t)=0
$$

Thus,

$$
\widehat{\mathcal{S}}(-t) \widehat{\mathcal{S}}(t)=I-\frac{1}{(p+1)^{2}} t^{2} \mathcal{D}_{s}(-t) \mathcal{D}_{s}(t)=I+\mathscr{O}\left(t^{2 p+2}\right),
$$

i.e., $\widehat{\mathcal{S}}$ is very close to selfadjoint. Now we reason in a similar way as in the proof of [4, Theorem II.3.2], proceeding from the local error structure (asymptotic expansion) for $\widehat{\mathcal{S}}(t)$,

$$
\widehat{\mathcal{S}}(t)=\mathcal{E}(t)+t^{p+2} C+\mathscr{O}\left(t^{p+3}\right) .
$$

Then,

$$
\begin{aligned}
I+\mathscr{O}\left(t^{2 p+2}\right) & =\widehat{\mathcal{S}}(-t) \widehat{\mathcal{S}}(t) \\
& =\left(\mathcal{E}(-t)+t^{p+2} C+\mathscr{O}\left(t^{p+3}\right)\right)\left(\mathcal{E}(t)+t^{p+2} C+\mathscr{O}\left(t^{p+3}\right)\right) \\
& =\underbrace{\mathcal{E}(-t) \mathcal{E}(t)}_{=I}+t^{p+2}(\mathcal{E}(-t) C+C \mathcal{E}(t))+\mathscr{O}\left(t^{p+3}\right) .
\end{aligned}
$$

Due to $\mathcal{E}( \pm t)=I \pm t H+\mathscr{O}\left(t^{2}\right)$ this implies $C=0$.

Theorem 1 also implies that the symmetrized defect-based local error estimator is of a better asymptotic quality than the classical one, with a deviation $\tilde{\mathcal{L}}(t)-\mathcal{L}(t)=\mathscr{O}\left(t^{p+3}\right)$ and not only $\mathscr{O}\left(t^{p+2}\right)$.

Remark 1. An inspection of the proof of Theorem 1 shows that the argument remains valid under the weaker assumption

$$
\mathcal{S}(-t) \mathcal{S}(t)=I+\mathscr{O}\left(t^{q}\right) \text { with } q>p+2 .
$$

This also shows that Theorem 1 can be applied to the corrected scheme $\widehat{\mathcal{S}}(t)$, with order $p+2 \geq 4$ instead of p, satisfying (see proof above)

$$
\widehat{\mathcal{S}}(-t) \widehat{\mathcal{S}}(t)=I+\mathscr{O}\left(t^{q}\right) \quad \text { with } q=2 p+2,
$$

since for $p \geq 4$ we have $q=2 p+2>(p+2)+2$, and therefore (8) is satisfied (mutatis mutandis) for the scheme of order $p+2$. This argument can be repeated inductively.

However, this method of increasing the order by defect correction will usually not be practically very relevant due to a prohibitive computational effort as compared to a straightforward higher-order method.

Remark 2. A modification of the approach presented here also applies to nonlinear and/or nonautonomous problems. Its practical efficiency remains to be investigated, however, and corresponding results and applications will be reported elsewhere.

\section{Splitting methods}

For problems (1) with a partitioned operator $H=A+B$, exponential splitting methods are commonly used because of their favorable computational properties $[1,2,3,4]$. As an example to illustrate the general considerations above, we resort to a symmetric 3 -stage splitting method

$$
\mathcal{S}=\mathcal{S}(t)=\mathrm{e}^{t A_{1}} \mathrm{e}^{t B_{1}} \mathrm{e}^{t A_{2}} \mathrm{e}^{t B_{1}} \mathrm{e}^{t A_{1}}
$$

where we denote $A_{j}=a_{j} A, B_{j}=b_{j} B$. For a consistent scheme we have $2 A_{1}+A_{2}=A$ and $2 B_{1}=B$. 
Practical evaluation of the defect. In this situation, the defect can be evaluated efficiently, see [2]: With

$$
v_{1}=\mathrm{e}^{t B_{1}} \mathrm{e}^{t A_{1}} u, \quad v_{2}=\mathrm{e}^{t B_{1}} \mathrm{e}^{t A_{2}} v_{1}, \quad v_{3}=\mathrm{e}^{t A_{1}} v_{2},
$$

the classical defect (3) evaluates to

$$
\begin{aligned}
\mathcal{D}_{c} u= & \mathrm{e}^{t A_{1}}\left(B_{1} v_{2}+\mathrm{e}^{t B_{1}} \mathrm{e}^{t A_{2}}\left(\left(A_{2}+B_{1}\right) v_{1}+\mathrm{e}^{t B_{1}} \mathrm{e}^{t A_{1}} A_{1} u\right)\right) \\
& -\left(A_{1}+A_{2}+B\right) v_{3} .
\end{aligned}
$$

Similarly for the symmetrized defect (5),

$$
\begin{aligned}
\mathcal{D}_{s} u= & \mathrm{e}^{t A_{1}}\left(B_{1} v_{2}+\mathrm{e}^{t B_{1}} \mathrm{e}^{t A_{2}}\left(\left(A_{2}+B_{1}\right) v_{1}+\mathrm{e}^{t B_{1}} \mathrm{e}^{t A_{1}}\left(-\left(\frac{1}{2} A_{2}+B_{1}\right) u\right)\right)\right) \\
& -\frac{1}{2}\left(A_{2}+B\right) v_{3} .
\end{aligned}
$$

Thus, the additional effort for the evaluation of $\mathcal{D}_{s} u$ only amounts to one more matrix-vector product. For an arbitrary number of stages the procedure is analogous.

\section{Numerical example}

To give a numerical illustration of the above considerations, we solve a linear Schrödinger equation

$$
\mathrm{i} \partial_{t} \psi(x, t)=-\frac{1}{2} \partial_{x}^{2} \psi(x, t)+V(x) \psi(x, t),
$$

with harmonic potential

$$
V(x)=\frac{1}{2} \omega^{2} x^{2},
$$

and exact solution

$$
\psi(x, t)=\left(\frac{\omega}{\pi}\right)^{\frac{1}{4}} \exp \left(-\frac{\omega}{2}\left(\mathrm{i} t+x^{2}\right)\right) .
$$

We choose $\omega=1$ and impose periodic boundary conditions on the interval $x \in[-H, H]$ with $H=10$. We use the 5 -stage 4th-order splitting scheme 'Emb 4/3 AKs' $(p=4)$ from the collection [5], with the potential $V(x)$ playing the role of $A$ and the kinetic term playing the role of $B$. The latter is discretized in space using pseudospectral Fourier-type collocation using 512 subintervals. Table 1 shows the local splitting error after a single step with stepsize $t$ together with the deviation of the local error estimates based on the classical and symmetrized defect, respectively.

\begin{tabular}{|c||c|c||c|c||c|c|}
\hline$t$ & $\left\|\mathcal{L} \psi_{0}\right\|_{2}$ & order & $\left\|\tilde{\mathcal{L}}_{c} \psi_{0}-\mathcal{L} \psi_{0}\right\|_{2}$ & order & $\left\|\tilde{\mathcal{L}}_{s} \psi_{0}-\mathcal{L} \psi_{0}\right\|_{2}$ & order \\
\hline \hline 0.40 & $1.77 \mathrm{e}-05$ & & $1.42 \mathrm{e}-06$ & & $6.31 \mathrm{e}-08$ & \\
0.20 & $5.57 \mathrm{e}-07$ & 5.0 & $2.23 \mathrm{e}-08$ & 6.0 & $4.95 \mathrm{e}-10$ & 7.0 \\
0.10 & $1.74 \mathrm{e}-08$ & 5.0 & $3.49 \mathrm{e}-10$ & 6.0 & $3.87 \mathrm{e}-12$ & 7.0 \\
0.05 & $5.45 \mathrm{e}-10$ & 5.0 & $5.45 \mathrm{e}-12$ & 6.0 & $3.16 \mathrm{e}-14$ & 7.0 \\
\hline
\end{tabular}

Table 1: Numerical results for example (10). The norm $\|\cdot\|_{2}$ is gauged in a way such that it is a discrete analog of the $L_{2}$-norm on $[-H, H]$.

\section{Acknowledgements}

This work was supported in part by the Vienna Science and Technology Fund (WWTF) [grant number MA14-002] and the Austrian Science Fund (FWF) [grant number P 30819-N32].

We are grateful to Daniel Haberlik, student at TU Wien, for performing numerical experiments. 


\section{References}

[1] W. Auzinger, O. Koch, M. Thalhammer, Defect-based local error estimators for splitting methods, with application to Schrödinger equations, Part I: The linear case, J. Comput. Appl. Math. 236 (2012) 2643-2659.

[2] W. Auzinger, O. Koch, M. Thalhammer, Defect-based local error estimators for splitting methods, with application to Schrödinger equations, Part II: Higher-order methods for linear problems, J. Comput. Appl. Math. 255 (2013) $384-403$.

[3] W. Auzinger, H. Hofstätter, O. Koch, M. Thalhammer, Defect-based local error estimators for splitting methods, with application to Schrödinger equations, Part III: The nonlinear case, J. Comput. Appl. Math. 273 (2014) $182-204$.

[4] E. Hairer, C. Lubich, G. Wanner, Geometric Numerical Integration, Springer-Verlag, Berlin-Heidelberg-New York, 2002.

[5] W. Auzinger, H. Hofstätter, O. Koch, Coefficients of various splitting methods,

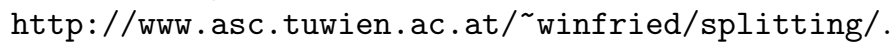

